\title{
Spinal Myoclonus As a Rare Presentation of Neurological Disease in Sudan
}

Corresponding Author:

Etedal Ahmed A. Ibrahim;

The National Center for

Neurological Science ,Al

Neelain University, Khartoum,

Sudan.

Postal code: 11111

email:

eetedalibrahim@yahoo.com

Received 22 January 2020

Accepted 24 May 2020

Published 30 June 2020

Production and Hosting by

Knowledge $\mathrm{E}$

(c) Etedal Ahmed A. Ibrahim and Asmhan M. Osman. This article is distributed under the terms of the Creative

Commons Attribution

License, which permits unrestricted use and redistribution provided that the original author and source are credited.

Editor-in-Chief:

Prof. Mohammad A. M. Ibnouf

\author{
Etedal Ahmed A. Ibrahim, ${ }^{1,2}$ and Asmhan M. Osman²,3 \\ ${ }^{1}$ Alneelain University, Khartoum, Sudan \\ ${ }^{2}$ The National Center for Neurological Science, Khartoum, Sudan \\ ${ }^{3}$ Nile Valley University, Atbara, Sudan
}

\section{Abstract}

Background: Spinal myoclonus is a very rare movement disorder characterized by myoclonic involvement of the whole body. Structural lesions are usually the cause, however, in primary spinal myoclonus, the etiology remains unknown.

Case description: We report a case of 50 year-old farmer from the eastern part of Sudan presented with jerky movement affecting his entire body, and diagnosed clinically with a spinal myoclonus. Laboratory study was normal and the MRI of brain and cervical and dorsal spines were also normal. The patient received clonazepam with marked improvement.

Conclusion: In any case of spinal myoclonus, EEG, EMG, and MRI of brain and spinal cord must be done to exclude structural lesions. Clonazepam is the drug of choice with significant improvement. In segmental spinal myoclonus, botulinum toxin is the best treatment.

Keywords: Spinal myoclonus, Sudan

\section{Introduction}

Myoclonusis a movement disorder presented with sudden, brief, shock-like jerks. Most myoclonic jerks are due to a brief burst of muscular activity, resulting in positive myoclonus [1]. Jerks resulting from brief cessation of ongoing muscular activity are called negative myoclonus (NM). While positive myoclonus is generally more common, NM frequently occurs in hospital settings as a result of toxic metabolic causes. A combination of both forms may be present in one disease, as in post-hypoxic myoclonus or progressive myoclonic epilepsies (PMEs).

\section{Spinal myoclonus}

Spinal myoclonus may be segmental or propriospinal, reflecting spinal segmental organization and the presence of propriospinal pathways that connect different spinal seg- 
may persist in sleep) or voluntary action (therefore it is present at rest, without activation), and may or may not be stimulus-sensitive [3]. Spinal segmental myoclonus is usually symptomatic of an underlying structural lesion such as syringomyelia, myelitis, spinal cord trauma, vascular lesion, or malignancy [2]. It is confined to one or few contiguous myotomes and may occur irregularly or quasi-rhythmically, with a frequency as low as $12 /$ min or as high as $100 / \mathrm{min}$. EMG myoclonic bursts are prolonged up to $1000 \mathrm{~ms}$. Propriospinal myoclonus is a form of spinal myoclonus where the spinal generator recruits axial muscles up and down the spinal cord via long propriospinal pathways [2]. Typically, there are axial flexion jerks involving the neck, trunk, and hips with a frequency of $\mathrm{Hz}$. EMG bursts are long, lasting several $100 \mathrm{~ms}$. Clinically, it can be distinguished from brainstem myoclonus, which is also axial in distribution, by sparing of the face and insensitivity to auditory stimuli. It typically occurs spontaneously, especially in recumbent position, or may be provoked by tapping of the abdomen or by eliciting tendon reflexes. As opposed to segmental myoclonus, most patients with propriospinal myoclonus have no clear etiology. Symptomatic forms are reported in cervical trauma, tumor, or viral myelitis (brown, psychogenic forms of propriospinal myoclonus are now increasingly recognized) [4]. One recent study on a large cohort of patient with idiopathic spinal myoclonus showed that at least $30 \%$ of patients had a definite premovement potential, indicating that the etiology was psychogenic [5]. In another large series, a psychogenic cause was suggested in 34 out of 35 patients with axial jerks, who were initially thought to have propriospinal myoclonus [6]. To approach a patient with spinal myoclonus, we need a detailed history and examination of the exact cause and other associated diseases.

Investigation needed EEG, EMG showed specific myoclonic burst. Brain and spinal MRI to exclude structural lesion.

\section{Treatment of spinal myoclonus}

In spinal myoclonus, pharmacological treatment is unsatisfactory. Clonazepam is the first choice of drug for both types of spinal myoclonus, and dosages up to $6 \mathrm{mg}$ are needed to diminish spinal segmental myoclonus. Levetiracetam was reported to be effective in a series of three patients with spinal segmental myoclonus [7].

\section{Case Description}

A 50-year-old man from the eastern part of Sudan presented with an abnormal movement that suddenly started affecting his entire body-a sudden jerky, rhythmic movement, similar to an electric shock, unaffected by light or noise. It was neither associated with convulsion nor with the loss of consciousness or headache, not even weakness or 


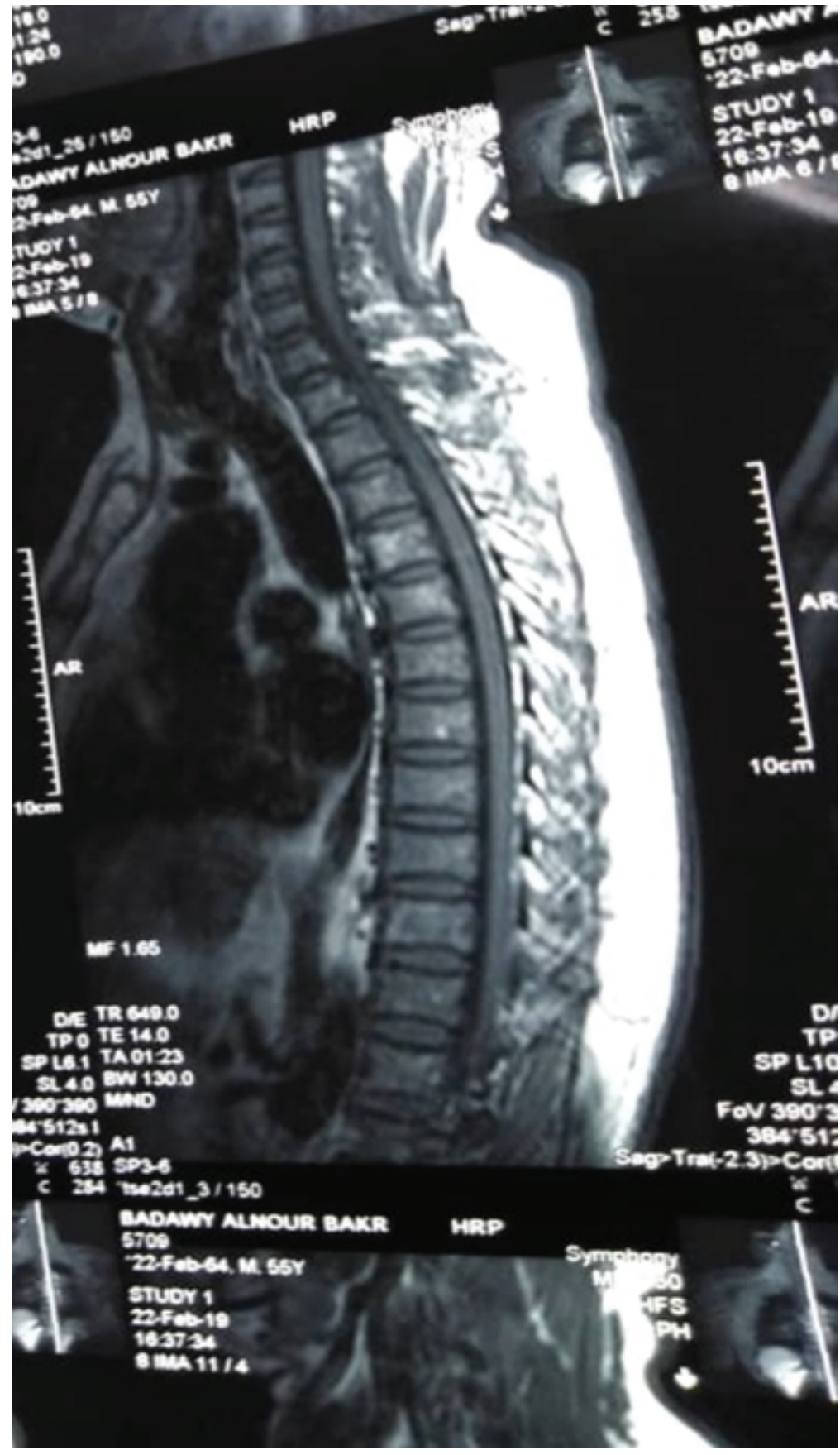

Figure 1: Cervical MRI and dorsal MRI.

sensory disturbance, the patient could not walk because of abnormal movement. The sphincter was controlled and a systemic review was non-significant; he had suffered two attacks in 2012-2015 with the same abnormal movement that were aborted by medication. There was no record of patient's family and drug history. O/E exam the patient not pale fully conscious oriented. CVS, chest and Abdomen were normal. Neurologically, he was fully conscious, with normal Mini-Mental score test, normal tone and power. Reflexes in the upper and lower limbs, planter were flexor, with mildly impaired pass pointing and normal sensation. The jerky movement of head, trunk, upper 

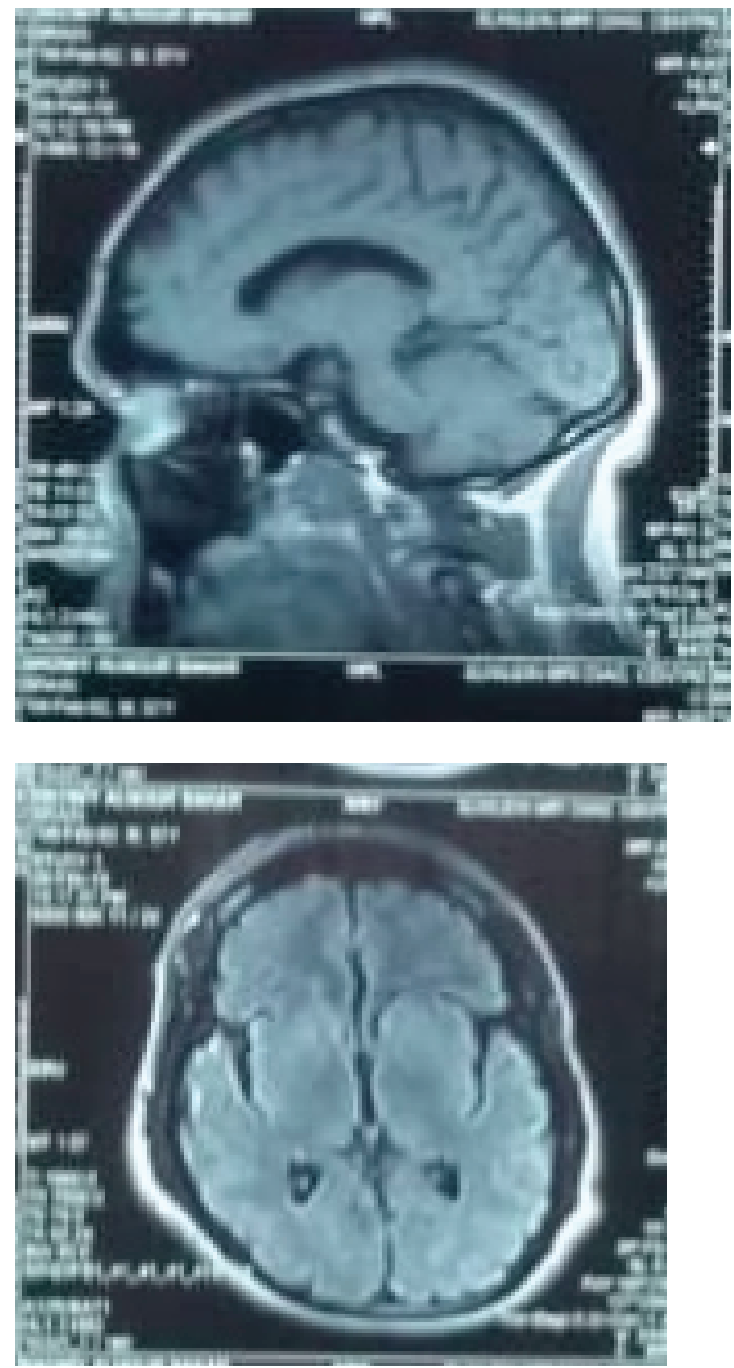

Figure 2: Brain MRI.

and lower limbs increased when changing posture, was unaffected by noise or light, no opsoclonus was seen, and the movement could not be suppressed by patient's will. He could not walk because the movement interfered with his normal walking. General investigations were normal, brain and spinal cord MRI, EEG, and EMG were normal (Figures 1 and 2). The patient received clonazepam $4 \mathrm{mg}$ with remarkable improvement, he started to walk without aid and the movement was totally aborted. He was discharged and sent home in a good condition.

\section{Discussion}

The current case is presented with unusual and distinct form of jerks, clinically compatible with manifestation of spinal myoclonus. Normal brain and spinal MRI exclude structural lesions. There was no ataxia or certain history of toxin, nor a significant family history of spinal myoclonus. We noticed that myoclonic jerk in the current case were 
spontaneous and not sensitive to external stimuli; this feature basically excludes the possibility of reticular reflex myoclonus that is usually stimulus-sensitive. The significant exacerbation of myoclonic symptoms before sleep in the current case was noticeable in 1997; Matagna et al. first described that spinal myoclonus can arise when a patient is mentally and physically relaxed, particularly close to the onset of sleep. They further discovered that jerks occurred when alpha activities on EEG were recorded.

\section{Conclusion}

In any case of spinal myoclonus, EEG, EMG, and brain and spinal cord MRI must be done to exclude structural lesions. The best treatment is clonazepam with significant improvement. In segmental spinal myoclonus, botulinum toxin is the best treatment.

\section{References}

[1] Shibasaki, H. and Hallett, M. (2005). Electrophysiological studies of myoclonus. Muscle Nerve, vol. 31, no. 2, pp. 157-174.

[2] Brown, P., Rothwell, J. C., Thompson, P. D., et al. (1994). Propriospinal myoclonus: evidence for spinal "pattern" generators in humans. Movement Disorders, vol. 9, no. 5, pp. 571-576.

[3] Caviness, J. N. and Brown, P. (2004). Myoclonus: current concepts and recent advances. The Lancet Neurology, vol. 3, no.10, pp. 598-607.

[4] Williams, D. R., Cowey, M., Tuck, K., et al. (2008). Psychogenic propriospinal myoclonus. Movement Disorders, vol. 23, no. 9, pp. 1312-1313.

[5] Esposito, M., Edwards, M. J., Bhatia, K. P., et al. (2009). Idiopathic spinal myoclonus: a clinical and neurophysiological assessment of a movement disorder of uncertain origin. Movement Disorders, vol. 24, no. 16, pp. 2344-2349.

[6] van der Salm, S. M., Koelman, J. H., Henneke, S., et al. (2010). Axial jerks: a clinical spectrum ranging from propriospinal to psychogenic myoclonus. Journal of Neurology, vol. 257, no. 8, pp. 1349-1355.

[7] Keswani, S. C., Kossoff, E. H., Krauss, G. L., et al. (2002). Amelioration of spinal myoclonus with levetiracetam. Journal of Neurology, Neurosurgery, and Psychiatry, vol. 73 , no. 5, pp. 457-458. 